\title{
Parlamentarisches Fragerecht: lediglich übersichtlicher Einstieg
}

\author{
Brenner, Michael: Reichweite und Grenzen des parlamentarischen Fragerechts, Nomos Verlags- \\ gesellschaft, Baden-Baden 2009, 80 Seiten, € 22,-.
}

Das Fragerecht ist in der parlamentarischen Demokratie von zentraler Bedeutung; es dient dazu, den Abgeordneten die zur Ausübung ihres Mandats erforderlichen Informationen zu verschaffen, wobei es allerdings immer wieder zu Unsicherheiten über den genauen Umfang dieses Rechts, auch Interpellationsrecht genannt, kommt.

Michael Brenners Arbeit will diesen Schwierigkeiten begegnen. Sie geht auf sein im Auftrag des Thüringer Innenministeriums erstattetes Gutachten zurück, dessen Anlass 652 gleichlautende Kleine Anfragen unterschiedlicher Abgeordneter zum Zeitpunkt des Inkrafttretens von Straßenausbaubeitragssatzungen waren, die sich lediglich hinsichtlich der Gemeinden, auf die sie sich bezogen, unterschieden. Leider beschränkt sich die Studie in weiten Teilen auf die Fragestellungen dieses Gutachtens und konzentriert sich dabei insbesondere auf die thüringische Rechtslage, so dass sie, um es vorwegzunehmen, ihrem Anspruch, „Reichweite und Grenzen des parlamentarischen Fragerechts“ zu beleuchten, nur teilweise gerecht wird.

Zutreffend geht Brenner davon aus, dass das parlamentarische Fragerecht grundsätzlich mit einer entsprechenden Verpflichtung der Regierung zur Beantwortung gestellter Fragen korrespondiere (S. 11). Soweit er in diesem Zusammenhang allerdings - ohne jedweden Beleg - behauptet, die Verfassung von Bremen enthielte eine Verpflichtung der Landesregierung, entsprechende Anfragen zu beantworten (S. 11), kann ihm nicht zugestimmt werden, da sich dem Wortlaut des Art. 100 BremLV, der das Fragerecht der Abgeordneten regelt, eine solche Verpflichtung (im Gegensatz zu Art. 105 Abs. 4 BremLV) gerade nicht entnehmen lässt.

Im Folgenden untersucht Brenner schwerpunktmäßig die Grenzen des Fragerechts, wobei er einleitend konstatiert, dass „das Informationsrecht der Abgeordneten zahlreichen Einschränkungen sowohl durch das Verfassungsrecht als auch durch das Geschäftsordnungsrecht unterworfen " sei (S. 21); letztere stellt er jedoch nicht erschöpfend dar, insbesondere fehlt eine Auseinandersetzung mit dem Beschluss des Niedersächsischen Staatsgerichtshofs vom 17. Januar 2008, demzufolge der „Schutzbereich“ des verfassungsrechtlichen Fragerechts durch die Geschäftsordnung des Parlaments bestimmt werden soll. ${ }^{1}$ Zutreffend führt der Autor aber aus, dass das Fragerecht ,auch inneren, ungeschriebenen Grenzen unterworfen“ sei (S. 22). Soweit er dies dahingehend konkretisiert, dass dieses Recht „nur gerichtet sein [könne] auf Bereiche, für die die Regierung Verantwortung trägt“ (S. 22), übersieht er allerdings, dass das Fragerecht landesverfassungsrechtlich durchaus unterschiedlich ausgestaltet ist und etwa in Hamburg die Abgeordneten berechtigt sind, ,in öffentlichen Angelegenheiten" Anfragen an den Senat zu richten (Art. 25 Abs. $1 \mathrm{HmbLV}$ ), wozu nach zutreffender Ansicht des Hamburgischen Verfassungsgerichts auch Angelegenheiten außerhalb des Bereichs der Regierungsverantwortung gehören können. ${ }^{2}$

Hinsichtlich der Antwortpflicht der Regierung differenziert Brenner danach, ob überhaupt eine Antwort gegeben werden muss und wie diese gegebenenfalls zu erfolgen hat (S. 32).

2 HmbVerfG, HmbJVBl. 1978, S. 11 ff., S. 15; HmbVerfG, LVerfGE 14, S. 221 ff., S. 227. 
Auch hier stellt er eingehend die thüringische Rechtslage dar (S. 30 bis 37) und führt aus, Art. 67 Abs. 3 ThürLV begrenze die Möglichkeit zur Antwortverweigerung nicht abschließend (S. 34); zudem habe die Landesregierung im Anwendungsbereich des - dem Wortlaut nach als Kann-Bestimmung ausgestalteten - Art. 67 Abs. 3 S. 1 ThürLV in aller Regel sogar die Pflicht, eine Auskunft jedenfalls nicht öffentlich zu erteilen, da anderenfalls in unzulässiger Weise in gegenläufige verfassungsrechtliche Positionen eingegriffen würde (S. 37). Brenner kommt dabei zu dem Ergebnis, letztlich seien „alle privaten wie auch alle öffentlichen (Staats-)Geheimnisse vor einer Veröffentlichung im Wege einer Regierungsantwort auf eine parlamentarische Anfrage geschützt" (S. 38), wobei seine Ausführungen allerdings eine Auseinandersetzung mit der Entscheidung des Bayerischen Verfassungsgerichtshofs vom 26. Juli 2006 vermissen lassen. Dieser hatte festgestellt, dass die unterschiedlichen Interessen einander im Wege der praktischen Konkordanz so zugeordnet werden müssen, dass beide soweit wie möglich ihre Wirkungen entfalten und das landesverfassungsrechtliche Fragerecht durch bundesrechtlich geregelte Geheimhaltungspflichten nicht zwangsläufig überlagert wird. ${ }^{3}$

Sodann erörtert Brenner eingehend die nicht nur geringfügige Beeinträchtigung der Funktionsfähigkeit der Landesregierung als Grenze einer Antwortpflicht, wobei er zutreffend annimmt, dass die Regierung die Bindung von Arbeitskapazitäten durch die Beantwortung parlamentarischer Anfragen einkalkulieren und sich gegebenenfalls auch auf Anfragen in großer Zahl und mit umfangreicher Thematik einstellen müsse (S. 43); wenn die parlamentarischen Anfragen ihrer Zahl oder ihrem Inhalt nach allerdings die Lahmlegung der Regierung bezweckten oder bewirkten, könne eine Antwortpflicht entfallen (S. 44). Zweifelhaft erscheint hingegen der in diesem Zusammenhang pauschal erhobene - aus Art. 67 Abs. 1 Nr. 2 ThürLV abgeleitete - Befund, „im Konfliktfall [... habe] die Funktionsfähigkeit der Regierung Vorrang gegenüber der Beantwortung einer parlamentarischen Anfrage“ (S. 46). Im Weiteren untersucht Brenner den so genannten Kernbereich exekutiver Eigenverantwortung als Grenze einer Antwortpflicht, wobei er die Landesregierung im Falle einer erheblichen Beeinträchtigung dieses Bereichs sogar als zur Auskunftsverweigerung verpflichtet ansieht (S. 48).

Abschließend beleuchtet er die gerichtliche Überprüfbarkeit des Antwortverhaltens der Exekutive, der er insoweit eine Einschätzungsprärogative zubilligt: Die Einschätzung der Regierung sowohl über das „Ob“ als auch das „Wie“ der Beantwortung einer parlamentarischen Frage könne verfassungsgerichtlich „nicht in allen Einzelheiten“ nachgeprüft werden (S. 70, vgl. auch S. 40), wobei jedoch offen bleibt, wo der Autor genau die Grenze ziehen will.

Zusammenfassend lässt sich festhalten, dass die für die parlamentarischen Informationsrechte in Thüringen bedeutsame Arbeit die Rechtslage in den anderen Bundesländern leider nur am Rande behandelt und auch die Auswertung der Rechtsprechung und Literatur nicht erschöpfend erfolgt - selbst die grundlegende Arbeit von Christian Teuber bleibt unberücksichtigt. ${ }^{4}$ Gleichwohl liefert die vorliegende Monographie einen übersichtlichen Einstieg in das Interpellationsrecht.

\section{Sebastian Berger} len des Bundestages und des Landtages Nordrhein-Westfalen, Berlin 2007, siehe auch die Rezension dieses Bandes von Julia von Blumenthal in diesem Heft der ZParl. 